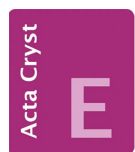

CRYSTALLOGRAPHIC COMMUNICATIONS

ISSN 2056-9890

Received 14 July 2020

Accepted 3 August 2020

Edited by L. Van Meervelt, Katholieke Universiteit Leuven, Belgium

Keywords: benzothiazinones; nitrobenzamides; anti-tuberculosis drugs; reaction mechanism; crystal structure.

CCDC reference: 2021003

Supporting information: this article has supporting information at journals.iucr.org/e
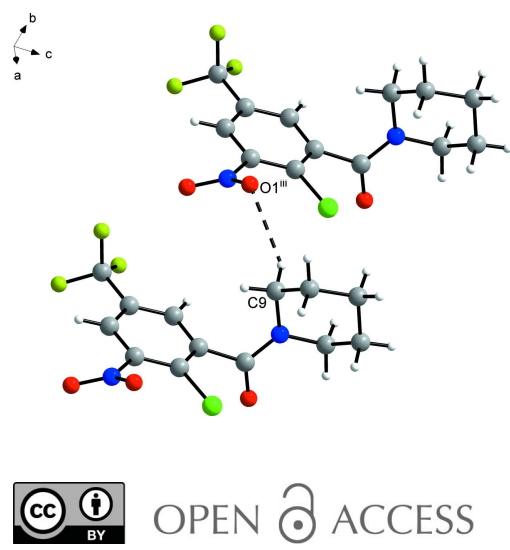

\section{[2-Chloro-3-nitro-5-(trifluoromethyl)phenyl](piper- idin-1-yl)methanone: structural characterization of a side product in benzothiazinone synthesis}

\author{
Tamira Eckhardt, ${ }^{\text {a }}$ Richard Goddard, ${ }^{\mathrm{b}}$ Ines Rudolph, ${ }^{\mathrm{a}}$ Adrian Richter, ${ }^{\mathrm{a}}$ Christoph \\ Lehmann, ${ }^{\text {a }}$ Peter Imming ${ }^{a}$ and Rüdiger W. Seidel ${ }^{\mathrm{a} *}$
}

aInstitut für Pharmazie, Wolfgang-Langenbeck-Str. 4, 06120 Halle (Saale), Germany, and bax-Planck-Institut für
Kohlenforschung, Kaiser-Wilhelm-Platz 1, 45470 Mülheim an der Ruhr, Germany. ${ }^{*}$ Correspondence e-mail:
ruediger.seidel@pharmazie.uni-halle.de

1,3-Benzothiazin-4-ones (BTZs) are a promising new class of anti-tuberculosis drug candidates, some of which have reached clinical trials. The title compound, the benzamide derivative [2-chloro-3-nitro-5-(trifluoromethyl)phenyl](piperidin-1-yl)methanone, $\mathrm{C}_{13} \mathrm{H}_{12} \mathrm{ClF}_{3} \mathrm{~N}_{2} \mathrm{O}_{3}$, occurs as a side product as a result of competitive reaction pathways in the nucleophilic attack during the synthesis of the BTZ 8-nitro-2-(piperidin-1-yl)-6-(trifluoromethyl)-1,3-benzothiazin-4-one, following the original synthetic route, whereby the corresponding benzoyl isothiocyanate is reacted with piperidine as secondary amine. In the title compound, the nitro group and the nearly planar amide group are significantly twisted out of the plane of the benzene ring. The piperidine ring adopts a chair conformation. The trifluoromethyl group exhibits slight rotational disorder with a refined ratio of occupancies of 0.972 (2):0.028 (2). There is structural evidence for intermolecular weak $\mathrm{C}-\mathrm{H} \cdots \mathrm{O}$ hydrogen bonds.

\section{Chemical context}

1,3-Benzothiazin-4-ones (BTZs) are promising anti-tuberculosis drug candidates, some of which have already reached clinical trials (Mikušová et al., 2014; Makarov \& Mikušová, 2020). Various methods for the synthesis of BTZs have been reported (Makarov et al., 2007; Moellmann et al., 2009; Makarov, 2011; Rudolph, 2014; Rudolph et al., 2016; Zhang \& Aldrich, 2019). In the original synthesis, 2-chlorobenzoyl chloride derivatives are reacted with ammonium or alkali metal thiocyanates to form the corresponding 2-chlorobenzoyl isothiocyanates (Makarov et al., 2007; Moellmann et al., 2009). These are reactive species and are treated in situ with secondary amines to afford the corresponding thiourea derivatives, which undergo ring closure to give 1,3-thiazin-4ones via an intramolecular nucleophilic substitution reaction. The latter step is favoured when electron-withdrawing substituents are present on the benzene ring.<smiles>O=C(c1cc(C(F)(F)F)cc([N+](=O)[O-])c1Cl)N1CCCCC1</smiles> 


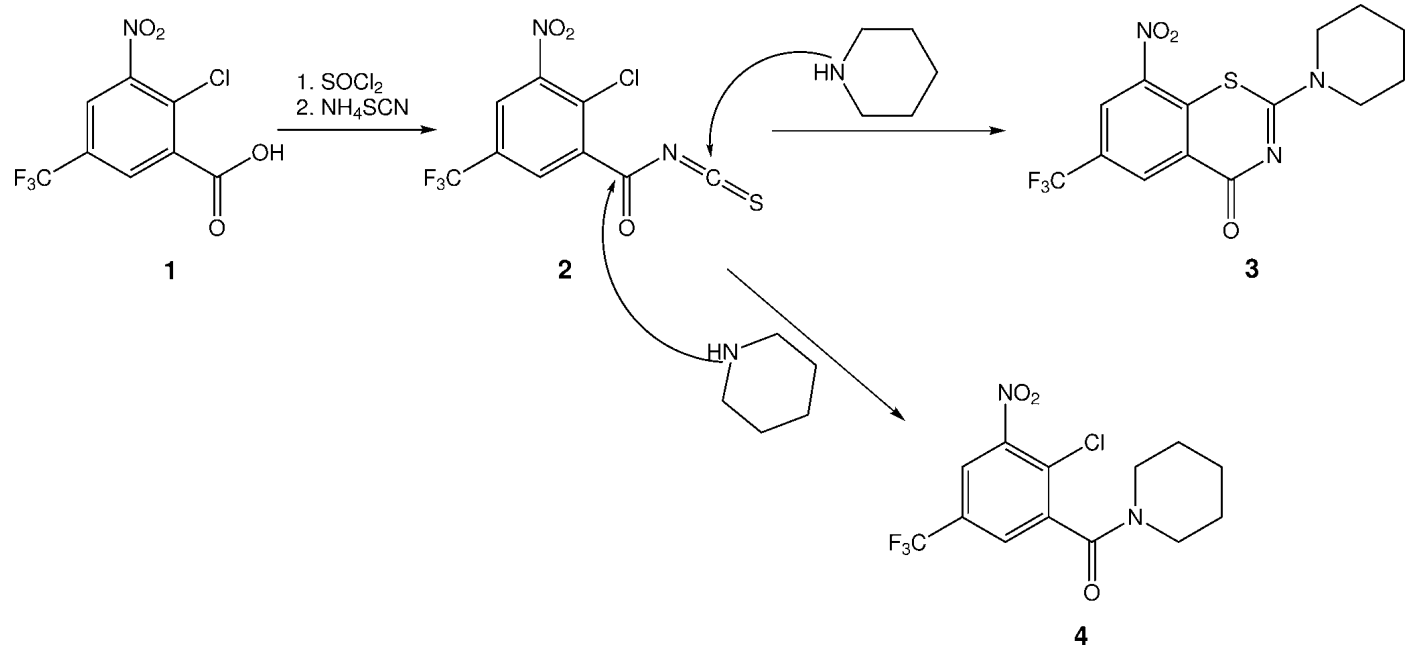

Figure 1

Synthetic pathway from 2-chloro-3-nitro-5-(trifluoromethyl)benzoic acid (1) to BTZ $\mathbf{3}$ and side product $\mathbf{4}$, illustrating the two different points of nucleophilic attack of piperidine at the intermediate 2-chloro-3-nitro-5-(trifluoromethyl)benzoyl isothiocyanate (2), resulting in 3 and 4 (Rudolph, 2014).

Fig. 1 depicts the synthesis following the original procedure for a BTZ previously reported by us (Rudolph, 2014; Rudolph et al., 2016; Richter, Rudolph et al., 2018). After treatment of 2-chloro-3-nitro-5-(trifluoromethyl)benzoic acid (1) with thionyl chloride and subsequently ammonium thiocyanate, the corresponding 2-chloro-3-nitro-5-(trifluoromethyl)benzoyl isothiocyanate (2) was reacted with piperidine. As illustrated, nucleophilic attack of the piperidine nitrogen atom at the isothiocyanate carbon atom leads to the anticipated 8-nitro2-(piperidin-1-yl)-6-(trifluoromethyl)-1,3-benzothiazin-4-one (3). The alternative nucleophilic attack at the carbonyl carbon atom affords the side product (2-chloro-3-nitro-5-(trifluoromethyl)phenyl)(piperidin-1-yl)methanone (4), which was structurally characterized by X-ray crystallography in the present work. The ratio of $\mathbf{3}$ to $\mathbf{4}$ was found to vary depending on the reaction conditions. Temperatures at or below $283 \mathrm{~K}$ favour the formation of the anticipated $\mathbf{3}$, whereas substantial amounts of $\mathbf{4}$ form at elevated temperatures (Rudolph, 2014). Since BTZs are in clinical development [see, for example, Makarov \& Mikušová (2020) or Mariandyshev et al. (2020)], this observation is not only important for the improvement of synthetic yields but also for the compilation of known synthetic side products for drug quality control.

It is interesting to note that dinitrobenzamide derivatives related to $\mathbf{4}$ have been found to have some anti-mycobacterial activity (Christophe et al., 2009; Trefzer et al., 2010; Tiwari et al., 2013), and the non-chlorinated analogue of $\mathbf{4}$ was reported to have anticoccidial activity (Welch et al., 1969).

\section{Structural commentary}

Fig. 2 shows the molecular structure of $\mathbf{4}$ in the solid state. Selected geometric parameters are listed in Table 1. The dihedral angle between the plane of the nitro group and the mean plane of the benzene ring is $38.1(2)^{\circ}$, which can be attributed to the steric demand of the neighbouring chloro substituent at the benzene ring. The trifluoromethyl group exhibits rotational disorder over two sites with $97.2(2) \%$ occupancy for the major site. The plane of the amide group, as defined by $\mathrm{C} 8, \mathrm{O} 3$ and $\mathrm{N} 2$, is tilted out of the mean plane of the benzene ring by $79.6(1)^{\circ}$. The Winkler-Dunitz parameters for the amide linkage $\tau$ (twist angle) $=1.2^{\circ}$ and $\chi_{\mathrm{N}}$ (pyramidalization at nitrogen $)=4.0^{\circ}$ indicate an almost planar amide group (Winkler \& Dunitz, 1971). In the IR spectrum (see supporting information), the band at $1639 \mathrm{~cm}^{-1}$ can be assigned to the $\mathrm{C}=\mathrm{O}$ stretching vibration of the amide group. The molecule is axially chiral, although the centrosymmetric crystal structure contains both enantiomers. The ${ }^{13} \mathrm{C}$ NMR spectrum of $\mathbf{4}$ in methanol- $d_{4}$ as well as chloroform- $d$ at room temperature (see supporting information) displays five distinct signals in the aliphatic region, which are assigned to the piperidine carbon atoms, indicating that the rotation about the amide $\mathrm{C}-\mathrm{N}$ bond is slow in solution under these conditions. The ${ }^{13} \mathrm{C}$ NMR chemical shift of the $\alpha$-carbon atom $\mathrm{C} 13$ syn to

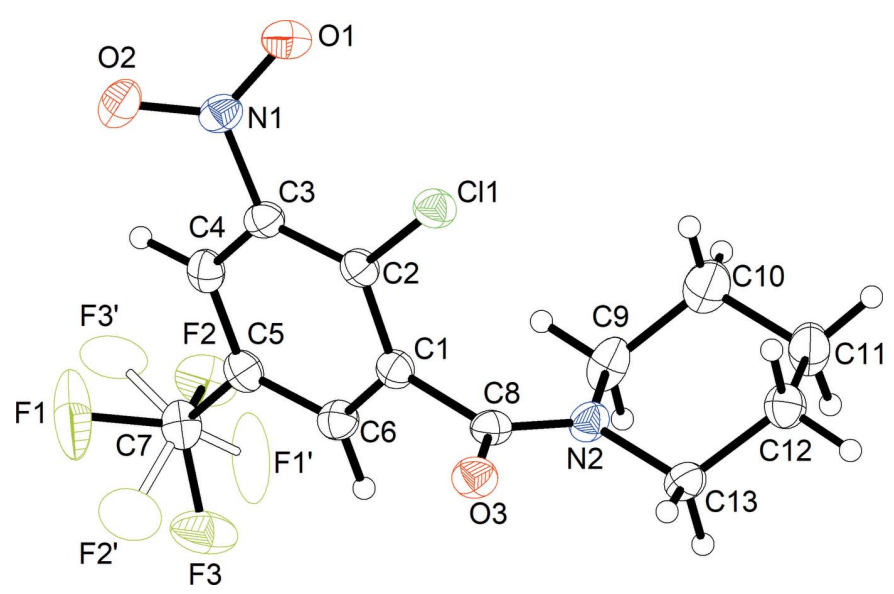

Figure 2

Molecular structure of 4. Displacement ellipsoids are drawn at the $50 \%$ probability level. $\mathrm{H}$ atoms are represented by small spheres of arbitrary radii. The minor occupancy component of the disordered trifluoromethyl group is depicted by empty ellipsoids. 
Table 1

Selected geometric parameters $\left(\AA{ }^{\circ}\right)$.

\begin{tabular}{lclr}
\hline C1-C8 & $1.510(3)$ & C7-F3 & $1.328(3)$ \\
C2-Cl1 & $1.725(2)$ & C7-F2 & $1.336(3)$ \\
C3-N1 & $1.468(3)$ & C8-O3 & $1.234(2)$ \\
C5-C7 & $1.497(3)$ & C8-N2 & $1.342(3)$ \\
C7-F1 & $1.325(3)$ & & \\
& & & \\
C4-C3-N1 & $116.41(17)$ & N2-C9-C10 & $110.59(18)$ \\
C2-C3-N1 & $122.35(18)$ & C9-C10-C11 & $110.61(19)$ \\
F1-C7-F3 & $107.69(19)$ & C12-C11-C10 & $109.74(18)$ \\
F1-C7-F2 & $105.98(19)$ & C13-C12-C11 & $111.01(18)$ \\
F3-C7-F2 & $105.59(17)$ & N2-C13-C12 & $111.35(17)$ \\
F1-C7-C5 & $112.43(17)$ & O2-N1-O1 & $124.48(17)$ \\
F3-C7-C5 & $112.80(18)$ & O2-N1-C3 & $117.04(17)$ \\
F2-C7-C5 & $111.86(17)$ & O1-N1-C3 & $118.44(16)$ \\
O3-C8-N2 & $124.72(18)$ & C8-N2-C13 & $120.26(16)$ \\
O3-C8-C1 & $118.43(18)$ & C8-N2-C9 & $124.74(16)$ \\
N2-C8-C1 & $116.85(17)$ & C13-N2-C9 & $114.89(16)$ \\
& & & \\
C4-C3-N1-O2 & $36.6(2)$ & O3-C8-N2-C13 & $3.0(3)$ \\
C2-C3-N1-O2 & $-143.29(19)$ & C1-C8-N2-C13 & $-176.62(17)$ \\
C4-C3-N1-O1 & $-141.34(18)$ & O3-C8-N2-C9 & $179.0(2)$ \\
C2-C3-N1-O1 & $38.8(3)$ & C1-C8-N2-C9 & $-0.6(3)$ \\
\hline
\end{tabular}

the carbonyl oxygen atom of the amide group is shielded compared with that of the anti $\alpha$-carbon atom $\mathrm{C} 9$. In chloroform- $d$, the observed shielding magnitude of $\Delta \delta_{\mathrm{C}}=5.0 \mathrm{ppm}$ is within the range expected for a benzoylpiperidine (Rubiralta et al., 1991). In the corresponding ${ }^{1} \mathrm{H}$ NMR spectrum, the syn protons with respect to the amide carbonyl oxygen atom are deshielded compared with those in the anti position $\left(\Delta \delta_{\mathrm{H}}=\right.$ $0.58 \mathrm{ppm})$. Complete assignments of ${ }^{1} \mathrm{H}$ and ${ }^{13} \mathrm{C}$ NMR data in chloroform- $d$ by ${ }^{13} \mathrm{C}, \mathrm{H}-\mathrm{HSQC}$ and -HMBC NMR spectra can be found in the supporting information. Notably, the two separated methylene ${ }^{1} \mathrm{H}$ NMR signals assigned to $\mathrm{C} 10$ in chloroform- $d$ appear as one signal in methanol- $d_{4}$.

In the solid state, the piperidine ring in $\mathbf{4}$ adopts a lowenergy chair conformation with some minor angular deviations from ideal tetrahedral values, resulting from planarity at N2 due to involvement in the amide linkage. The puckering parameters of the piperidine six-membered ring, as calculated with PLATON (Spek, 2020), are $Q=0.555$ (2) $\AA, \theta=4.1(2)^{\circ}$ and $\varphi=161(3)^{\circ}$. By way of comparison, the total puckering amplitude $Q$ is $0.63 \AA$ and the magnitude of distortion $\theta$ is $0^{\circ}$ for an ideal cyclohexane chair (Cremer \& Pople, 1975).

\section{Supramolecular features}

In general, the crystal structure of $\mathbf{4}$ appears to be dominated by close packing. According to Kitaigorodskii (1973), the space group $\mathrm{Pbca}$ is among those available for the densest packing of molecules of arbitrary shape. Nevertheless, the solid-state supramolecular structure features $\mathrm{C}-\mathrm{H} \cdots \mathrm{O}$ contacts between an aromatic $\mathrm{CH}$ moiety and the amide oxygen atom of an adjacent molecule (Fig. $3 a$ ). The corresponding geometric parameters (Table 2) support the interpretation as a weak hydrogen bond (Thakuria et al., 2017). These interactions link the molecules into strands extending by $2_{1}$ screw symmetry in the [010] direction. The $\alpha$-methylene groups of the piperidine ring, on which the amide group
Table 2

Hydrogen-bond geometry $\left(\AA,^{\circ}\right)$.

\begin{tabular}{lllll}
\hline$D-\mathrm{H} \cdots A$ & $D-\mathrm{H}$ & $\mathrm{H} \cdots A$ & $D \cdots A$ & $D-\mathrm{H} \cdots A$ \\
\hline $\mathrm{C}^{-}-\mathrm{H} 6 \cdots \mathrm{O} 3^{\text {i }}$ & 0.95 & 2.59 & $3.526(3)$ & 169 \\
$\mathrm{C}^{\mathrm{ii}}-\mathrm{H} 9 A \cdots \mathrm{O} 1^{\text {ii }}$ & 0.99 & 2.45 & $3.361(3)$ & 154 \\
$\mathrm{C} 9-\mathrm{H} 9 B \cdots \mathrm{O} 1^{\text {iii }}$ & 0.99 & 2.58 & $3.369(3)$ & 137 \\
$\mathrm{C} 13-\mathrm{H} 13 A \cdots C g(\mathrm{C} 1-\mathrm{C} 6)^{\text {iv }}$ & 0.99 & 2.92 & $3.447(2)$ & 114 \\
\hline
\end{tabular}

Symmetry codes: (i) $-x+1, y+\frac{1}{2},-z+\frac{1}{2}$; (ii) $-x+\frac{1}{2}, y+\frac{1}{2}, z$; (iii) $x, y+1, z$; (iv) $x+\frac{3}{2},-y+\frac{1}{2},-z$.

should exert an electron-withdrawing effect, also form intermolecular $\mathrm{C}-\mathrm{H} \cdots \mathrm{O}$ and $\mathrm{C}-\mathrm{H} \cdots \pi$ contacts, respectively, to the nitro group and the benzene ring of adjacent molecules (Fig. $3 b-d$ ). The corresponding geometric parameters (Table 2), however, reveal that these contacts may not have the same significance here as the aforementioned $\mathrm{C}_{\text {aromatic }}-$ $\mathrm{H} \cdot \mathrm{O}_{\text {amide }}$ short contact (Wood et al., 2009). It is also worth noting that $\pi-\pi$ stacking of the aromatic rings is not observed.

\section{Database survey}

A search of the Cambridge Structural Database (CSD; version 5.41 with March 2020 updates; Groom et al., 2016) for related substituted $N$-benzoyl-piperidine compounds revealed about 30 structures, of which (2-chloro-3,5-dinitrophenyl)(piperidin1-yl)methanone (CSD refcode: URALIJ; Luo et al., 2011) is structurally most related to 4 . Similar to $\mathbf{4}$, the 3-nitro group with the neighbouring chloro substituent is tilted out of the mean plane of the benzene ring by $36.2^{\circ}$. At $75.8^{\circ}$, the dihedral angle between the amide plane and the mean plane of the benzene ring is comparable with that in 4. Likewise, the
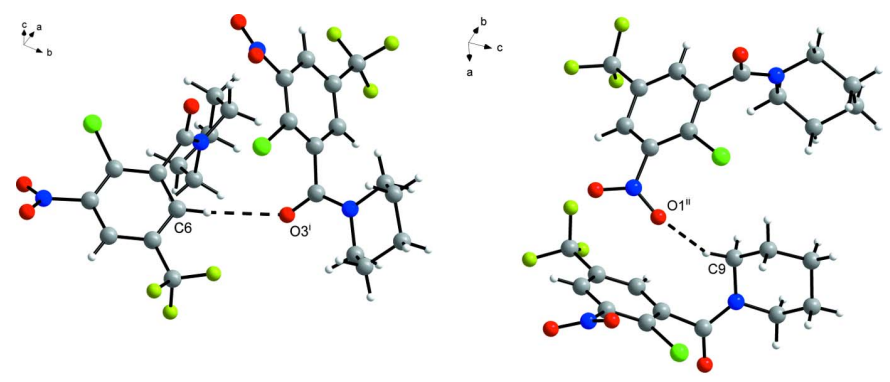

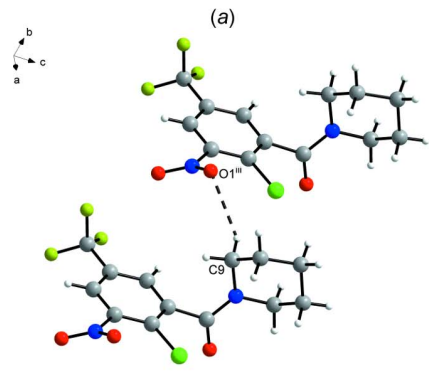

(c)

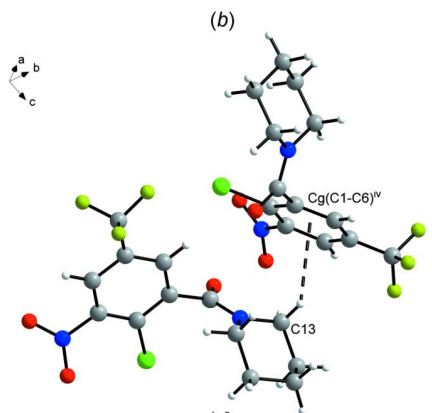

(d)
Figure 3

Short contacts (dashed lines) between adjacent molecules in the crystal structure of 4 . The minor component of the disordered trifluoromethyl group is omitted for clarity. Symmetry codes: (i) $-x+1, y+\frac{1}{2},-z+\frac{1}{2}$; (ii) $-x+\frac{1}{2}, y+\frac{1}{2}, z$; (iii) $x, y+1, z$; (iv) $x+\frac{3}{2},-y+\frac{1}{2},-z$. 
piperidine ring exhibits a chair conformation with a planar structure at the nitrogen atom. In contrast to $\mathbf{4}$, the solid-state supramolecular structure of URALIJ exhibits $\pi-\pi$ stacking of the aromatic rings. Interestingly, a CSD search for the 2-chloro-3-nitro-5-(trifluoromethyl)phenyl moiety present in $\mathbf{4}$ led to only one structure, viz. 2-chloro-1,3-dinitro-5-(trifluoromethyl)benzene (JIHNUM; del Casino et al., 2018), also known as chloralin, which is active against Plasmodium, but which also shows toxicity in mice.

\section{Anti-mycobacterial evaluation}

The anti-mycobacterial activity of $\mathbf{4}$ was evaluated against Mycobacterium smegmatis $\mathrm{mc}^{2} 155$ and Mycobacterium abscessus ATCC19977, using broth microdilution assays [for the assay protocols, see the supporting information and Richter, Strauch et al. (2018)]. For both mycobacterial species, no growth inhibition was detectable up to a concentration of $100 \mu \mathrm{M}$. For M. smegmatis, the findings are consistent with the activity data for a related nitrobenzamide derivative reported by Tiwari et al. (2013). CT319, a 3-nitro-5-(trifluoromethyl)benzamide derivative, however, showed activity against $M$. smegmatis $\mathrm{mc}^{2} 155$ and other mycobacterial strains (Trefzer $e t$ al., 2010).

\section{Synthesis and crystallization}

Chemicals were purchased and used as received. The synthesis of $\mathbf{1}$ is described elsewhere (Welch et al., 1969). Solvents were of reagent grade and were distilled before use. The IR spectrum was measured on a Bruker TENSOR II FT-IR spectrometer at a resolution of $4 \mathrm{~cm}^{-1}$. NMR spectra were recorded at room temperature on an Agilent Technologies VNMRS $400 \mathrm{MHz}$ NMR spectrometer (abbreviations: $d=$ doublet, $q=$ quartet, $m=$ multiplet). Chemical shifts are referenced to the residual signals of methanol- $d_{4}\left(\delta_{\mathrm{H}}=3.35\right.$ $\left.\mathrm{ppm}, \delta_{\mathrm{C}}=49.3 \mathrm{ppm}\right)$ or chloroform- $d\left(\delta_{\mathrm{H}}=7.26 \mathrm{ppm}, \delta_{\mathrm{C}}=77.2\right.$ ppm).

$2.7 \mathrm{~mL}(37.0 \mathrm{mmol})$ of $\mathrm{SOCl}_{2}$ were added to a stirred solution of $\mathbf{1}(5.00 \mathrm{~g}, 18.5 \mathrm{mmol})$ in toluene, and the mixture was heated to reflux for two h. The solvent was subsequently removed under reduced pressure, and the acid chloride thus obtained was used without purification. The residue was taken up in $6.5 \mathrm{~mL}$ of acetonitrile and a solution of $1.41 \mathrm{~g}$ (18.5 mmol) $\mathrm{NH}_{4} \mathrm{SCN}$ in $55 \mathrm{~mL}$ of acetonitrile was added dropwise with stirring to obtain 2 in situ. After stirring for $5 \mathrm{~min}$ at $313 \mathrm{~K}$, the resulting $\mathrm{NH}_{4} \mathrm{Cl}$ precipitate was filtered off, and $3.7 \mathrm{~mL}(37.0 \mathrm{mmol})$ of piperidine were added. The mixture was refluxed overnight, and then the solvent was removed under reduced pressure. Water was added to the residue and, after extraction with dichloromethane, the organic phase was washed with $10 \%$ aqueous $\mathrm{NaHCO}_{3}$ and dried over $\mathrm{MgSO}_{4}$. After removal of the solvent, the crude product was subjected to flash chromatography on silica gel, eluting with ethyl acetate $/ n$-heptane (gradient $10-50 \% \mathrm{v} / \mathrm{v}$ ), to isolate $1.09 \mathrm{~g}(3.0 \mathrm{mmol}, 16 \%)$ of $\mathbf{3}$ and a minor amount of the side product $4 .{ }^{1} \mathrm{H}$ and ${ }^{13} \mathrm{C}$ NMR spectroscopic and mass
Table 3

Experimental details.

\begin{tabular}{ll}
\hline Crystal data & \\
Chemical formula & $\mathrm{C}_{13} \mathrm{H}_{12} \mathrm{ClF}_{3} \mathrm{~N}_{2} \mathrm{O}_{3}$ \\
$M_{\mathrm{r}}$ & 336.70 \\
Crystal system, space group & Orthorhombic, Pbca \\
Temperature (K) & 100 \\
$a, b, c(\AA)$ & $18.0904(7), 7.8971(3), 19.8043(8)$ \\
$V\left(\AA^{3}\right)$ & $2829.28(19)$ \\
$Z$ & 8 \\
Radiation type & $\mathrm{Cu} \mathrm{K \alpha}$ \\
$\mu\left(\mathrm{mm}^{-1}\right)$ & 2.88 \\
Crystal size $(\mathrm{mm})$ & $0.59 \times 0.50 \times 0.44$
\end{tabular}

Data collection

Diffractometer

Absorption correction

$T_{\min }, T_{\max }$

No. of measured, independent and observed $[I>2 \sigma(I)]$ reflections

$R_{\text {int }}$

$(\sin \theta / \lambda)_{\max }\left(\AA^{-1}\right)$

Bruker Kappa Mach3 APEXII

Gaussian (SADABS; Krause et al., 2015)

$0.297,0.586$

49954, 2784, 2699

0.041

0.617

Refinement

$R\left[F^{2}>2 \sigma\left(F^{2}\right)\right], w R\left(F^{2}\right), S$

No. of reflections

No. of parameters

No. of restraints

$\mathrm{H}$-atom treatment

$\Delta \rho_{\max }, \Delta \rho_{\min }\left(\mathrm{e} \AA^{-3}\right)$

$0.043,0.115,1.15$
2784
209
45
H-atom parameters constrained
$0.32,-0.32$

Computer programs: APEX3 (Bruker, 2017), SAINT (Bruker, 2004), SHELXT2014/4 (Sheldrick, 2015a), SHELXL2018/3 (Sheldrick, 2015b), DIAMOND (Brandenburg, 2018), enCIFer (Allen et al., 2004) and publCIF (Westrip, 2010).

spectrometric data of $\mathbf{3}$ were in agreement with those in the literature (Rudolph, 2014; Rudolph et al., 2016). Crystals of 4 suitable for X-ray crystallography were obtained from a solution in ethyl acetate/heptane (1:1) by slow evaporation of the solvents at room temperature. NMR spectroscopic data for 4:

${ }^{1} \mathrm{H}$ NMR $\left(400 \mathrm{MHz}, \mathrm{CD}_{3} \mathrm{OD}\right) \delta 8.42\left(d,{ }^{4} J_{\text {meta }}=2.2 \mathrm{~Hz}, 1 \mathrm{H}\right.$, $\mathrm{Ar}-H), 8.09\left(d,{ }^{4} J_{\text {meta }}=2.2 \mathrm{~Hz}, 1 \mathrm{H}, \mathrm{Ar}-H\right), 3.88-3.71(\mathrm{~m}$, $\left.2 \mathrm{H}, \mathrm{N}-\mathrm{CH}_{2}\right), 3.33-3.21\left(m, 2 \mathrm{H}, \mathrm{N}-\mathrm{CH}_{2}\right), 1.76\left(m, 4 \mathrm{H}, \mathrm{CH}_{2}\right)$, $1.64\left(m, 2 \mathrm{H}, \mathrm{CH}_{2}\right) \mathrm{ppm} ;{ }^{13} \mathrm{C}$ NMR $\left(101 \mathrm{MHz}, \mathrm{CD}_{3} \mathrm{OD}\right) \delta 165.5$, $150.7,141.8,132.3\left(q,{ }^{2} J_{\mathrm{C}, \mathrm{F}}=35 \mathrm{~Hz}\right), 129.2\left(q,{ }^{3} J_{\mathrm{C}, \mathrm{F}}=4 \mathrm{~Hz}\right)$, 128.1, $124.4\left(q,{ }^{3} J_{\mathrm{C}, \mathrm{F}}=4 \mathrm{~Hz}\right), 124.1\left(q,{ }^{1} J_{\mathrm{C}, \mathrm{F}}=273 \mathrm{~Hz}\right), 49.5$, 44.3, 27.6, 26.7, $25.5 \mathrm{ppm}$.

${ }^{1} \mathrm{H}$ NMR $\left(400 \mathrm{MHz}, \mathrm{CDCl}_{3} \delta\right) 8.07\left(d,{ }^{4} J_{\text {meta }}=2.0 \mathrm{~Hz}, 1 \mathrm{H}\right.$, $\mathrm{C} 4-H), 7.73\left(d,{ }^{4} J_{\text {meta }}=2.0 \mathrm{~Hz}, 1 \mathrm{H}, \mathrm{C} 6-H\right), 3.83-3.68(\mathrm{~m}$, $\left.2 \mathrm{H}, \mathrm{C} 13-\mathrm{CH}_{2}\right), 3.22\left(d d d,{ }^{2} J_{\mathrm{gem}}=13.2 \mathrm{~Hz},{ }^{3} J_{\mathrm{vic}}=7.1,4.0 \mathrm{~Hz}\right.$, $\left.1 \mathrm{H}, \mathrm{C} 9-\mathrm{CH}_{2}\right), 3.15\left(d d d,{ }^{2} J_{\mathrm{gem}}=13.2 \mathrm{~Hz},{ }^{3} J_{\mathrm{vic}}=7.1,4.0 \mathrm{~Hz}\right.$, $\left.1 \mathrm{H}, \mathrm{C} 9-\mathrm{CH}_{2}\right), 1.70\left(\mathrm{~m}, 4 \mathrm{H}, \mathrm{C} 11, \mathrm{C} 12-\mathrm{CH}_{2}\right), 1.65-1.57(\mathrm{~m}$, $\left.1 \mathrm{H}, \mathrm{C} 10-\mathrm{CH}_{2}\right), 1.56-1.47\left(m, 1 \mathrm{H}, \mathrm{C} 10-\mathrm{CH}_{2}\right) \mathrm{ppm} ;{ }^{13} \mathrm{C} \mathrm{NMR}$ $\left(101 \mathrm{MHz}, \mathrm{CDCl}_{3} \delta\right) 163.5(\mathrm{C} 8, \mathrm{C}=\mathrm{O}), 148.9(\mathrm{C} 3), 141.1(\mathrm{C} 1)$, $131.2\left(q,{ }^{2} J_{\mathrm{C}, \mathrm{F}}=35 \mathrm{~Hz}, \mathrm{C} 5\right), 127.8\left(q,{ }^{3} J_{\mathrm{C}, \mathrm{F}}=4 \mathrm{~Hz}, \mathrm{C} 6\right), 127.6$ (C2), $122.7\left(q,{ }^{3} J_{\mathrm{C}, \mathrm{F}}=4 \mathrm{~Hz}, \mathrm{C} 4\right), 122.4\left(q,{ }^{1} J_{\mathrm{C}, \mathrm{F}}=273 \mathrm{~Hz}, \mathrm{C} 7\right)$, 48.3 (C9), 43.3 (C13), 26.7 (C10), 25.7 (C12), 24.6 (C11) ppm.

\section{Refinement}

Crystal data, data collection and structure refinement details are summarized in Table 3. The rotational disorder of the trifluoromethyl group was refined using a split model with 
similar distance restraints on the 1,2- and 1,3-distances and equal atomic displacement parameters for opposite fluorine atoms belonging to different disorder sites. Refinement of the ratio of occupancies by means of a free variable resulted in 0.972 (2):0.028 (2). Hydrogen-atom positions were calculated geometrically with $\mathrm{C}_{\mathrm{a}}-\mathrm{H}=0.95 \AA$ and $\mathrm{C}_{\mathrm{m}}-\mathrm{H}=0.99 \AA(\mathrm{a}=$ aromatic and $\mathrm{m}=$ methylene), and refined with the appropriate riding model and $U_{\text {iso }}(\mathrm{H})=1.2 U_{\text {eq }}(\mathrm{C})$.

\section{Acknowledgements}

We thank Professor Christian W. Lehmann for providing access to the X-ray diffraction facility and Heike Schucht for technical assistance.

\section{Funding information}

We acknowledge the financial support within the funding programme Open Access Publishing by the German Research Foundation (DFG).

\section{References}

Allen, F. H., Johnson, O., Shields, G. P., Smith, B. R. \& Towler, M. (2004). J. Appl. Cryst. 37, 335-338.

Brandenburg, K. (2018). DIAMOND. Crystal Impact GbR, Bonn, Germany.

Bruker (2004). SAINT. Bruker AXS Inc., Madison, Wisconsin, USA. Bruker (2017). APEX3. Bruker AXS Inc., Madison, Wisconsin, USA.

Casino, A. del, Lukinović, V., Bhatt, R., Randle, L. E., Dascombe, M. J., Fennell, B. J., Drew, M. G. B., Bell, A., Fielding, A. J. \& Ismail, F. M. D. (2018). ChemistrySelect 3, 7572-7580.

Christophe, T., Jackson, M., Jeon, H. K., Fenistein, D., ContrerasDominguez, M., Kim, J., Genovesio, A., Carralot, J.-P., Ewann, F., Kim, E. H., Lee, S. Y., Kang, S., Seo, M. J., Park, E. J., Škovierová, H., Pham, H., Riccardi, G., Nam, J. Y., Marsollier, L., Kempf, M., Joly-Guillou, M.-L., Oh, T., Shin, W. K., No, Z., Nehrbass, U., Brosch, R., Cole, S. T. \& Brodin, P. (2009). PLoS Pathog. 5, e1000645.

Cremer, D. \& Pople, J. A. (1975). J. Am. Chem. Soc. 97, 13541358.

Groom, C. R., Bruno, I. J., Lightfoot, M. P. \& Ward, S. C. (2016). Acta Cryst. B72, 171-179.

Kitaigorodskii, A. I. (1973). Molecular Crystals and Molecules. London: Academic Press.
Krause, L., Herbst-Irmer, R., Sheldrick, G. M. \& Stalke, D. (2015). J. Appl. Cryst. 48, 3-10.

Luo, X., Huang, Y.-C., Gao, C. \& Yu, L.-T. (2011). Acta Cryst. E67, o1066.

Makarov, V. (2011). PCT Int. Appl. WO 2011132070 A1.

Makarov, V., Cole, S. T. \& Moellmann, U. (2007). PCT Int. Appl. WO 2007134625 A1.

Makarov, V. \& Mikušová, K. (2020). Appl. Sci. 10, 2269.

Mariandyshev, A. O., Khokhlov, A. L., Smerdin, S. V., Shcherbakova, V. S., Igumnova, O. V., Ozerova, I. V., Bolgarina, A. A. \& Nikitina, N. A. (2020). Ter. Arkh. 92, 61-72.

Mikušová, K., Makarov, V. \& Neres, J. (2014). Curr. Pharm. Des. 20, 4379-4403.

Moellmann, U., Makarov, V. \& Cole, S. T. (2009). PCT Int. Appl. WO 2009010163 A1.

Richter, A., Rudolph, I., Möllmann, U., Voigt, K., Chung, C., Singh, O. M. P., Rees, M., Mendoza-Losana, A., Bates, R., Ballell, L., Batt, S., Veerapen, N., Fütterer, K., Besra, G., Imming, P. \& Argyrou, A. (2018). Sci. Rep. 8, 13473.

Richter, A., Strauch, A., Chao, J., Ko, M. \& Av-Gay, Y. (2018). Antimicrob. Agents Chemother. 62, e00828-18.

Rubiralta, M., Giralt, E. \& Diez, A. (1991). 7 - N-Acylpiperidines. A Useful Tool for Stereocontrol in Organic Synthesis. In: Studies in Organic Chemistry, vol. 43, pp. 193-224. Amsterdam: Elsevier.

Rudolph, A. I. (2014). PhD thesis, Martin-Luther-Universität HalleWittenberg, Halle (Saale), Germany.

Rudolph, I., Imming, P. \& Richter, A. (2016). Ger. Offen. DE 102014012546 A1 20160331.

Sheldrick, G. M. (2015a). Acta Cryst. A71, 3-8.

Sheldrick, G. M. (2015b). Acta Cryst. C71, 3-8

Spek, A. L. (2020). Acta Cryst. E76, 1-11.

Thakuria, R., Sarma, B. \& Nangia, A. (2017). Hydrogen Bonding in Molecular Crystals. In: Comprehensive Supramolecular Chemistry II, vol. 7, edited by J. L. Atwood, pp. 25-48. Oxford: Elsevier.

Tiwari, R., Moraski, G. C., Krchňák, V., Miller, P. A., Colon-Martinez, M., Herrero, E., Oliver, A. G. \& Miller, M. J. (2013). J. Am. Chem. Soc. 135, 3539-3549.

Trefzer, C., Rengifo-Gonzalez, M., Hinner, M. J., Schneider, P., Makarov, V., Cole, S. T. \& Johnsson, K. (2010). J. Am. Chem. Soc. 132, 13663-13665.

Welch, D. E., Baron, R. R. \& Burton, B. A. (1969). J. Med. Chem. 12, 299-303.

Westrip, S. P. (2010). J. Appl. Cryst. 43, 920-925.

Winkler, F. K. \& Dunitz, J. D. (1971). J. Mol. Biol. 59, 169-182.

Wood, P. A., Allen, F. H. \& Pidcock, E. (2009). CrystEngComm, 11, $1563-1571$.

Zhang, G. \& Aldrich, C. C. (2019). Acta Cryst. C75, 1031-1035. 


\section{supporting information}

Acta Cryst. (2020). E76, 1442-1446 [https://doi.org/10.1107/S2056989020010658]

\section{[2-Chloro-3-nitro-5-(trifluoromethyl)phenyl] (piperidin-1-yl)methanone:} structural characterization of a side product in benzothiazinone synthesis

Tamira Eckhardt, Richard Goddard, Ines Rudolph, Adrian Richter, Christoph Lehmann, Peter Imming and Rüdiger W. Seidel

\section{Computing details}

Data collection: APEX3 (Bruker, 2017); cell refinement: SAINT (Bruker, 2004); data reduction: SAINT (Bruker, 2004); program(s) used to solve structure: SHELXT2014/4 (Sheldrick, 2015a); program(s) used to refine structure: SHELXL2018/3 (Sheldrick, 2015b); molecular graphics: DIAMOND (Brandenburg, 2018); software used to prepare material for publication: enCIFer (Allen et al., 2004) and publCIF (Westrip, 2010).

[2-Chloro-3-nitro-5-(trifluoromethyl)phenyl] (piperidin-1-yl)methanone

Crystal data

$\mathrm{C}_{13} \mathrm{H}_{12} \mathrm{ClF}_{3} \mathrm{~N}_{2} \mathrm{O}_{3}$

$M_{r}=336.70$

Orthorhombic, $\mathrm{Pbca}$

$a=18.0904$ (7) $\AA$

$b=7.8971(3) \AA$

$c=19.8043(8) \AA$

$V=2829.28(19) \AA^{3}$

$Z=8$

$F(000)=1376$

Data collection

Bruker Kappa Mach3 APEXII diffractometer

Radiation source: $0.2 \times 2 \mathrm{~mm}^{2}$ focus rotating anode

MONTEL graded multilayer optics monochromator

Detector resolution: 66.67 pixels $\mathrm{mm}^{-1}$ $\varphi$ - and $\omega$-scans

Absorption correction: gaussian

(SADABS; Krause et al., 2015)

Refinement

Refinement on $F^{2}$

Least-squares matrix: full

$R\left[F^{2}>2 \sigma\left(F^{2}\right)\right]=0.043$

$w R\left(F^{2}\right)=0.115$

$S=1.15$

2784 reflections

209 parameters
$D_{\mathrm{x}}=1.581 \mathrm{Mg} \mathrm{m}^{-3}$

$\mathrm{Cu} K \alpha$ radiation, $\lambda=1.54178 \AA$

Cell parameters from 9968 reflections

$\theta=4.9-71.6^{\circ}$

$\mu=2.88 \mathrm{~mm}^{-1}$

$T=100 \mathrm{~K}$

Block, colourless

$0.59 \times 0.50 \times 0.44 \mathrm{~mm}$

$T_{\min }=0.297, T_{\max }=0.586$

49954 measured reflections

2784 independent reflections

2699 reflections with $I>2 \sigma(I)$

$R_{\text {int }}=0.041$

$\theta_{\max }=72.2^{\circ}, \theta_{\min }=4.9^{\circ}$

$h=-21 \rightarrow 22$

$k=-9 \rightarrow 9$

$l=-24 \rightarrow 24$

45 restraints

Primary atom site location: dual

Secondary atom site location: difference Fourier map

Hydrogen site location: inferred from neighbouring sites

$\mathrm{H}$-atom parameters constrained 
$w=1 /\left[\sigma^{2}\left(F_{\mathrm{o}}^{2}\right)+(0.0508 P)^{2}+2.7994 P\right]$

where $P=\left(F_{\mathrm{o}}^{2}+2 F_{\mathrm{c}}^{2}\right) / 3$

$(\Delta / \sigma)_{\max }<0.001$

$$
\Delta \rho_{\max }=0.32 \mathrm{e} \AA^{-3}
$$

\section{Special details}

Geometry. All esds (except the esd in the dihedral angle between two 1.s. planes) are estimated using the full covariance matrix. The cell esds are taken into account individually in the estimation of esds in distances, angles and torsion angles; correlations between esds in cell parameters are only used when they are defined by crystal symmetry. An approximate (isotropic) treatment of cell esds is used for estimating esds involving 1.s. planes.

Fractional atomic coordinates and isotropic or equivalent isotropic displacement parameters $\left(\AA^{2}\right)$

\begin{tabular}{|c|c|c|c|c|c|}
\hline & $x$ & $y$ & $z$ & $U_{\text {iso }} * / U_{\text {eq }}$ & Occ. $(<1)$ \\
\hline $\mathrm{C} 1$ & $0.41556(10)$ & 0.3250 & $0.23581(10)$ & $0.0230(4)$ & \\
\hline $\mathrm{C} 2$ & $0.38370(10)$ & 0.1651 & $0.23833(10)$ & $0.0224(4)$ & \\
\hline $\mathrm{C} 3$ & $0.35007(10)$ & $0.1004(3)$ & $0.18023(10)$ & $0.0227(4)$ & \\
\hline $\mathrm{C} 4$ & $0.34763(10)$ & 0.1927 & $0.12111(10)$ & $0.0243(4)$ & \\
\hline $\mathrm{H} 4$ & 0.325215 & 0.146458 & 0.081847 & $0.029 *$ & \\
\hline $\mathrm{C} 5$ & $0.37831(10)$ & $0.3541(3)$ & $0.11961(10)$ & $0.0242(4)$ & \\
\hline C6 & $0.41190(11)$ & $0.4206(3)$ & $0.17666(10)$ & $0.0250(4)$ & \\
\hline H6 & 0.432417 & 0.531321 & 0.175487 & $0.030 *$ & \\
\hline $\mathrm{C} 7$ & $0.37425(12)$ & $0.4549(3)$ & $0.05572(11)$ & $0.0298(5)$ & \\
\hline $\mathrm{C} 8$ & $0.45848(10)$ & $0.3906(2)$ & $0.29575(10)$ & $0.0232(4)$ & \\
\hline $\mathrm{C} 9$ & $0.34929(13)$ & $0.5683(3)$ & $0.32422(11)$ & $0.0327(5)$ & \\
\hline H9A & 0.325497 & 0.503425 & 0.287381 & $0.039 *$ & \\
\hline H9B & 0.351745 & 0.688568 & 0.310277 & $0.039 *$ & \\
\hline C10 & $0.30335(12)$ & 0.5528 & $0.38813(13)$ & $0.0404(6)$ & \\
\hline $\mathrm{H} 10 \mathrm{~A}$ & 0.296350 & 0.431647 & 0.399304 & $0.048^{*}$ & \\
\hline H10B & 0.254021 & 0.603627 & 0.380559 & $0.048^{*}$ & \\
\hline C11 & $0.34134(13)$ & $0.6421(3)$ & $0.44689(11)$ & $0.0373(5)$ & \\
\hline H11A & 0.344826 & 0.764952 & 0.437412 & $0.045^{*}$ & \\
\hline H11B & 0.311898 & 0.626777 & 0.488581 & $0.045^{*}$ & \\
\hline $\mathrm{C} 12$ & $0.41843(12)$ & $0.5691(3)$ & $0.45695(10)$ & $0.0294(5)$ & \\
\hline $\mathrm{H} 12 \mathrm{~A}$ & 0.443955 & 0.632047 & 0.493365 & $0.035^{*}$ & \\
\hline $\mathrm{H} 12 \mathrm{~B}$ & 0.414464 & 0.449164 & 0.470978 & $0.035^{*}$ & \\
\hline C13 & $0.46343(11)$ & 0.5807 & $0.39245(10)$ & $0.0277(4)$ & \\
\hline H13A & 0.473754 & 0.701122 & 0.382205 & $0.033^{*}$ & \\
\hline H13B & 0.511333 & 0.522352 & 0.398982 & $0.033^{*}$ & \\
\hline N1 & $0.31581(9)$ & $-0.0681(2)$ & $0.17845(9)$ & $0.0253(4)$ & \\
\hline N2 & $0.42423(9)$ & $0.5038(2)$ & $0.33544(8)$ & $0.0249(4)$ & \\
\hline $\mathrm{O} 1$ & $0.28104(8)$ & $-0.1161(2)$ & $0.22819(8)$ & $0.0324(4)$ & \\
\hline $\mathrm{O} 2$ & $0.32229(8)$ & $-0.1493(2)$ & $0.12611(8)$ & $0.0341(4)$ & \\
\hline $\mathrm{O} 3$ & $0.52198(7)$ & 0.33806 (19) & $0.30471(7)$ & $0.0288(3)$ & \\
\hline $\mathrm{F} 1$ & $0.38438(14)$ & $0.3603(2)$ & $0.00122(7)$ & $0.0711(7)$ & $0.972(2)$ \\
\hline $\mathrm{F} 2$ & $0.30831(8)$ & 0.52847 (19) & $0.04797(8)$ & $0.0418(4)$ & $0.972(2)$ \\
\hline F3 & $0.42321(8)$ & $0.5801(2)$ & $0.05379(8)$ & $0.0470(4)$ & $0.972(2)$ \\
\hline $\mathrm{F} 1^{\prime}$ & $0.352(3)$ & $0.612(3)$ & $0.0688(18)$ & $0.0711(7)$ & $0.028(2)$ \\
\hline $\mathrm{F} 2^{\prime}$ & $0.4387(11)$ & $0.472(6)$ & $0.0269(18)$ & $0.0418(4)$ & $0.028(2)$ \\
\hline F3' & $0.325(2)$ & $0.397(5)$ & $0.0129(15)$ & $0.0470(4)$ & $0.028(2)$ \\
\hline
\end{tabular}




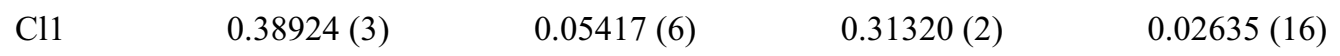

Atomic displacement parameters $\left(\AA^{2}\right)$

\begin{tabular}{lllllll}
\hline & $U^{11}$ & $U^{22}$ & $U^{33}$ & $U^{12}$ & $U^{13}$ & $U^{23}$ \\
\hline C1 & $0.0194(9)$ & $0.0250(9)$ & $0.0246(9)$ & $0.0021(7)$ & $0.0012(7)$ & $-0.0019(8)$ \\
C2 & $0.0186(8)$ & $0.0246(9)$ & $0.0239(9)$ & $0.0021(7)$ & $0.0011(7)$ & $0.0002(8)$ \\
C3 & $0.0166(8)$ & $0.0219(9)$ & $0.0296(10)$ & $0.0011(7)$ & $0.0009(7)$ & $-0.0021(8)$ \\
C4 & $0.0202(9)$ & $0.0288(10)$ & $0.0239(9)$ & $0.0050(8)$ & $-0.0004(7)$ & $-0.0031(8)$ \\
C5 & $0.0219(9)$ & $0.0261(10)$ & $0.0247(10)$ & $0.0056(8)$ & $0.0021(7)$ & $0.0011(8)$ \\
C6 & $0.0248(10)$ & $0.0226(9)$ & $0.0277(10)$ & $0.0006(8)$ & $0.0004(8)$ & $0.0002(8)$ \\
C7 & $0.0350(11)$ & $0.0292(11)$ & $0.0251(10)$ & $0.0056(9)$ & $0.0005(8)$ & $-0.0002(8)$ \\
C8 & $0.0225(9)$ & $0.0226(9)$ & $0.0245(9)$ & $-0.0035(8)$ & $-0.0003(7)$ & $0.0033(8)$ \\
C9 & $0.0335(11)$ & $0.0298(11)$ & $0.0347(11)$ & $0.0094(9)$ & $-0.0117(9)$ & $-0.0088(9)$ \\
C10 & $0.0200(10)$ & $0.0517(15)$ & $0.0495(14)$ & $0.0058(9)$ & $-0.0038(9)$ & $-0.0188(11)$ \\
C11 & $0.0324(11)$ & $0.0474(14)$ & $0.0322(11)$ & $0.0039(10)$ & $-0.0011(9)$ & $-0.0114(10)$ \\
C12 & $0.0308(11)$ & $0.0327(11)$ & $0.0247(10)$ & $-0.0010(9)$ & $-0.0013(8)$ & $0.0002(8)$ \\
C13 & $0.0267(10)$ & $0.0306(10)$ & $0.0257(10)$ & $-0.0070(8)$ & $-0.0028(8)$ & $-0.0018(8)$ \\
N1 & $0.0190(8)$ & $0.0260(9)$ & $0.0310(9)$ & $-0.0009(6)$ & $-0.0037(7)$ & $-0.0016(7)$ \\
N2 & $0.0213(8)$ & $0.0277(9)$ & $0.0257(8)$ & $-0.0010(7)$ & $-0.0042(7)$ & $-0.0030(7)$ \\
O1 & $0.0267(7)$ & $0.0334(8)$ & $0.0371(8)$ & $-0.0062(6)$ & $0.0010(6)$ & $0.0028(7)$ \\
O2 & $0.0348(8)$ & $0.0320(8)$ & $0.0356(8)$ & $-0.0010(6)$ & $-0.0045(6)$ & $-0.0098(7)$ \\
O3 & $0.0204(7)$ & $0.0324(8)$ & $0.0337(8)$ & $0.0005(6)$ & $-0.0017(6)$ & $-0.0003(6)$ \\
F1 & $0.151(2)$ & $0.0382(9)$ & $0.0239(7)$ & $0.0278(10)$ & $0.0154(9)$ & $-0.0002(6)$ \\
F2 & $0.0336(7)$ & $0.0457(8)$ & $0.0460(8)$ & $0.0053(6)$ & $-0.0056(6)$ & $0.0175(7)$ \\
F3 & $0.0389(8)$ & $0.0567(10)$ & $0.0453(8)$ & $-0.0142(7)$ & $-0.0052(6)$ & $0.0251(7)$ \\
F1' & $0.151(2)$ & $0.0382(9)$ & $0.0239(7)$ & $0.0278(10)$ & $0.0154(9)$ & $-0.0002(6)$ \\
F2 & $0.0336(7)$ & $0.0457(8)$ & $0.0460(8)$ & $0.0053(6)$ & $-0.0056(6)$ & $0.0175(7)$ \\
F3' & $0.0389(8)$ & $0.0567(10)$ & $0.0453(8)$ & $-0.0142(7)$ & $-0.0052(6)$ & $0.0251(7)$ \\
C11 & $0.0267(3)$ & $0.0273(3)$ & $0.0250(3)$ & $-0.00271(18)$ & $0.00002(17)$ & $0.00364(17)$ \\
& & & & & & \\
\hline & & & & & & \\
\hline
\end{tabular}

Geometric parameters $\left(\AA,{ }^{\circ}\right)$

\begin{tabular}{llll}
\hline $\mathrm{C} 1-\mathrm{C} 2$ & $1.389(3)$ & $\mathrm{C} 8-\mathrm{N} 2$ & $1.342(3)$ \\
$\mathrm{C} 1-\mathrm{C} 6$ & $1.395(3)$ & $\mathrm{C} 9-\mathrm{N} 2$ & $1.465(3)$ \\
$\mathrm{C} 1-\mathrm{C} 8$ & $1.510(3)$ & $\mathrm{C} 9-\mathrm{C} 10$ & $1.519(3)$ \\
$\mathrm{C} 2-\mathrm{C} 3$ & $1.398(3)$ & $\mathrm{C} 9-\mathrm{H} 9 \mathrm{~A}$ & 0.9900 \\
$\mathrm{C} 2-\mathrm{C} 11$ & $\mathrm{C} 9-\mathrm{H} 9 \mathrm{~B}$ & 0.9900 \\
$\mathrm{C} 3-\mathrm{C} 4$ & $1.725(2)$ & $\mathrm{C} 10-\mathrm{C} 11$ & $1.525(3)$ \\
$\mathrm{C} 3-\mathrm{N} 1$ & $1.380(3)$ & $\mathrm{C} 10-\mathrm{H} 10 \mathrm{~A}$ & 0.9900 \\
$\mathrm{C} 4-\mathrm{C} 5$ & $1.468(3)$ & $\mathrm{C} 10-\mathrm{H} 10 \mathrm{~B}$ & 0.9900 \\
$\mathrm{C} 4-\mathrm{H} 4$ & $1.390(3)$ & $\mathrm{C} 11-\mathrm{C} 12$ & $1.522(3)$ \\
$\mathrm{C} 5-\mathrm{C} 6$ & 0.9500 & $\mathrm{C} 11-\mathrm{H} 11 \mathrm{~A}$ & 0.9900 \\
$\mathrm{C} 5-\mathrm{C} 7$ & $1.386(3)$ & $\mathrm{C} 11-\mathrm{H} 11 \mathrm{~B}$ & 0.9900 \\
$\mathrm{C} 6-\mathrm{H} 6$ & $1.497(3)$ & $\mathrm{C} 12-\mathrm{C} 13$ & $1.518(3)$ \\
$\mathrm{C} 7-\mathrm{F} 2$ & $\mathrm{C} 12-\mathrm{H} 12 \mathrm{~A}$ & 0.9900 \\
$\mathrm{C} 7-\mathrm{F} 3$ & 1.9500 & $\mathrm{C} 12-\mathrm{H} 12 \mathrm{~B}$ & 0.9900 \\
$\mathrm{C} 7-\mathrm{F} 1$ & $1.314(14)$ & $\mathrm{C} 13-\mathrm{N} 2$ & $1.465(2)$
\end{tabular}




\begin{tabular}{|c|c|c|c|}
\hline $\mathrm{C} 7-\mathrm{F} 3$ & $1.328(3)$ & $\mathrm{C} 13-\mathrm{H} 13 \mathrm{~A}$ & 0.9900 \\
\hline $\mathrm{C} 7-\mathrm{F} 1^{\prime}$ & $1.332(14)$ & $\mathrm{C} 13-\mathrm{H} 13 \mathrm{~B}$ & 0.9900 \\
\hline $\mathrm{C} 7-\mathrm{F} 2$ & $1.336(3)$ & $\mathrm{N} 1-\mathrm{O} 2$ & $1.225(2)$ \\
\hline $\mathrm{C} 8-\mathrm{O} 3$ & $1.234(2)$ & $\mathrm{N} 1-\mathrm{O} 1$ & $1.229(2)$ \\
\hline $\mathrm{C} 2-\mathrm{C} 1-\mathrm{C} 6$ & $120.16(18)$ & $\mathrm{C} 10-\mathrm{C} 9-\mathrm{H} 9 \mathrm{~A}$ & 109.5 \\
\hline $\mathrm{C} 2-\mathrm{C} 1-\mathrm{C} 8$ & $119.82(17)$ & $\mathrm{N} 2-\mathrm{C} 9-\mathrm{H} 9 \mathrm{~B}$ & 109.5 \\
\hline $\mathrm{C} 6-\mathrm{C} 1-\mathrm{C} 8$ & $119.90(17)$ & $\mathrm{C} 10-\mathrm{C} 9-\mathrm{H} 9 \mathrm{~B}$ & 109.5 \\
\hline $\mathrm{C} 1-\mathrm{C} 2-\mathrm{C} 3$ & $118.92(18)$ & $\mathrm{H} 9 \mathrm{~A}-\mathrm{C} 9-\mathrm{H} 9 \mathrm{~B}$ & 108.1 \\
\hline $\mathrm{C} 1-\mathrm{C} 2-\mathrm{C} 11$ & $117.93(15)$ & $\mathrm{C} 9-\mathrm{C} 10-\mathrm{C} 11$ & $110.61(19)$ \\
\hline $\mathrm{C} 3-\mathrm{C} 2-\mathrm{C} 11$ & $123.13(16)$ & $\mathrm{C} 9-\mathrm{C} 10-\mathrm{H} 10 \mathrm{~A}$ & 109.5 \\
\hline $\mathrm{C} 4-\mathrm{C} 3-\mathrm{C} 2$ & $121.24(18)$ & $\mathrm{C} 11-\mathrm{C} 10-\mathrm{H} 10 \mathrm{~A}$ & 109.5 \\
\hline $\mathrm{C} 4-\mathrm{C} 3-\mathrm{N} 1$ & $116.41(17)$ & $\mathrm{C} 9-\mathrm{C} 10-\mathrm{H} 10 \mathrm{~B}$ & 109.5 \\
\hline $\mathrm{C} 2-\mathrm{C} 3-\mathrm{N} 1$ & $122.35(18)$ & $\mathrm{C} 11-\mathrm{C} 10-\mathrm{H} 10 \mathrm{~B}$ & 109.5 \\
\hline $\mathrm{C} 3-\mathrm{C} 4-\mathrm{C} 5$ & $119.32(18)$ & $\mathrm{H} 10 \mathrm{~A}-\mathrm{C} 10-\mathrm{H} 10 \mathrm{~B}$ & 108.1 \\
\hline $\mathrm{C} 3-\mathrm{C} 4-\mathrm{H} 4$ & 120.3 & $\mathrm{C} 12-\mathrm{C} 11-\mathrm{C} 10$ & $109.74(18)$ \\
\hline $\mathrm{C} 5-\mathrm{C} 4-\mathrm{H} 4$ & 120.3 & $\mathrm{C} 12-\mathrm{C} 11-\mathrm{H} 11 \mathrm{~A}$ & 109.7 \\
\hline $\mathrm{C} 6-\mathrm{C} 5-\mathrm{C} 4$ & $120.33(18)$ & $\mathrm{C} 10-\mathrm{C} 11-\mathrm{H} 11 \mathrm{~A}$ & 109.7 \\
\hline $\mathrm{C} 6-\mathrm{C} 5-\mathrm{C} 7$ & $120.58(19)$ & $\mathrm{C} 12-\mathrm{C} 11-\mathrm{H} 11 \mathrm{~B}$ & 109.7 \\
\hline $\mathrm{C} 4-\mathrm{C} 5-\mathrm{C} 7$ & $119.09(18)$ & $\mathrm{C} 10-\mathrm{C} 11-\mathrm{H} 11 \mathrm{~B}$ & 109.7 \\
\hline $\mathrm{C} 5-\mathrm{C} 6-\mathrm{C} 1$ & $119.98(19)$ & $\mathrm{H} 11 \mathrm{~A}-\mathrm{C} 11-\mathrm{H} 11 \mathrm{~B}$ & 108.2 \\
\hline $\mathrm{C} 5-\mathrm{C} 6-\mathrm{H} 6$ & 120.0 & $\mathrm{C} 13-\mathrm{C} 12-\mathrm{C} 11$ & $111.01(18)$ \\
\hline $\mathrm{C} 1-\mathrm{C} 6-\mathrm{H} 6$ & 120.0 & $\mathrm{C} 13-\mathrm{C} 12-\mathrm{H} 12 \mathrm{~A}$ & 109.4 \\
\hline $\mathrm{F} 2^{\prime}-\mathrm{C} 7-\mathrm{F} 3^{\prime}$ & $111.1(19)$ & $\mathrm{C} 11-\mathrm{C} 12-\mathrm{H} 12 \mathrm{~A}$ & 109.4 \\
\hline $\mathrm{F} 1-\mathrm{C} 7-\mathrm{F} 3$ & $107.69(19)$ & $\mathrm{C} 13-\mathrm{C} 12-\mathrm{H} 12 \mathrm{~B}$ & 109.4 \\
\hline $\mathrm{F} 2^{\prime}-\mathrm{C} 7-\mathrm{F} 1^{\prime}$ & $105.2(19)$ & $\mathrm{C} 11-\mathrm{C} 12-\mathrm{H} 12 \mathrm{~B}$ & 109.4 \\
\hline $\mathrm{F} 3^{\prime}-\mathrm{C} 7-\mathrm{F} 1^{\prime}$ & $104.0(19)$ & $\mathrm{H} 12 \mathrm{~A}-\mathrm{C} 12-\mathrm{H} 12 \mathrm{~B}$ & 108.0 \\
\hline $\mathrm{F} 1-\mathrm{C} 7-\mathrm{F} 2$ & $105.98(19)$ & $\mathrm{N} 2-\mathrm{C} 13-\mathrm{C} 12$ & $111.35(17)$ \\
\hline $\mathrm{F} 3-\mathrm{C} 7-\mathrm{F} 2$ & $105.59(17)$ & $\mathrm{N} 2-\mathrm{C} 13-\mathrm{H} 13 \mathrm{~A}$ & 109.4 \\
\hline $\mathrm{F} 2^{\prime}-\mathrm{C} 7-\mathrm{C} 5$ & $112.3(15)$ & $\mathrm{C} 12-\mathrm{C} 13-\mathrm{H} 13 \mathrm{~A}$ & 109.4 \\
\hline $\mathrm{F} 3{ }^{\prime}-\mathrm{C} 7-\mathrm{C} 5$ & $113.2(15)$ & $\mathrm{N} 2-\mathrm{C} 13-\mathrm{H} 13 \mathrm{~B}$ & 109.4 \\
\hline $\mathrm{F} 1-\mathrm{C} 7-\mathrm{C} 5$ & $112.43(17)$ & $\mathrm{C} 12-\mathrm{C} 13-\mathrm{H} 13 \mathrm{~B}$ & 109.4 \\
\hline $\mathrm{F} 3-\mathrm{C} 7-\mathrm{C} 5$ & $112.80(18)$ & $\mathrm{H} 13 \mathrm{~A}-\mathrm{C} 13-\mathrm{H} 13 \mathrm{~B}$ & 108.0 \\
\hline $\mathrm{F} 1{ }^{\prime}-\mathrm{C} 7-\mathrm{C} 5$ & $110.3(15)$ & $\mathrm{O} 2-\mathrm{N} 1-\mathrm{O} 1$ & $124.48(17)$ \\
\hline $\mathrm{F} 2-\mathrm{C} 7-\mathrm{C} 5$ & $111.86(17)$ & $\mathrm{O} 2-\mathrm{N} 1-\mathrm{C} 3$ & $117.04(17)$ \\
\hline $\mathrm{O} 3-\mathrm{C} 8-\mathrm{N} 2$ & $124.72(18)$ & $\mathrm{O} 1-\mathrm{N} 1-\mathrm{C} 3$ & $118.44(16)$ \\
\hline $\mathrm{O} 3-\mathrm{C} 8-\mathrm{C} 1$ & $118.43(18)$ & $\mathrm{C} 8-\mathrm{N} 2-\mathrm{C} 13$ & $120.26(16)$ \\
\hline $\mathrm{N} 2-\mathrm{C} 8-\mathrm{C} 1$ & $116.85(17)$ & $\mathrm{C} 8-\mathrm{N} 2-\mathrm{C} 9$ & $124.74(16)$ \\
\hline $\mathrm{N} 2-\mathrm{C} 9-\mathrm{C} 10$ & $110.59(18)$ & $\mathrm{C} 13-\mathrm{N} 2-\mathrm{C} 9$ & $114.89(16)$ \\
\hline $\mathrm{N} 2-\mathrm{C} 9-\mathrm{H} 9 \mathrm{~A}$ & 109.5 & & \\
\hline $\mathrm{C} 6-\mathrm{C} 1-\mathrm{C} 2-\mathrm{C} 3$ & $1.9(3)$ & $\mathrm{C} 6-\mathrm{C} 5-\mathrm{C} 7-\mathrm{F} 1^{\prime}$ & $-47(3)$ \\
\hline $\mathrm{C} 8-\mathrm{C} 1-\mathrm{C} 2-\mathrm{C} 3$ & $-174.16(17)$ & $\mathrm{C} 4-\mathrm{C} 5-\mathrm{C} 7-\mathrm{F} 1^{\prime}$ & $133(3)$ \\
\hline $\mathrm{C} 6-\mathrm{C} 1-\mathrm{C} 2-\mathrm{Cl}$ & $-179.32(15)$ & $\mathrm{C} 6-\mathrm{C} 5-\mathrm{C} 7-\mathrm{F} 2$ & $-99.4(2)$ \\
\hline $\mathrm{C} 8-\mathrm{C} 1-\mathrm{C} 2-\mathrm{C} 11$ & $4.6(2)$ & $\mathrm{C} 4-\mathrm{C} 5-\mathrm{C} 7-\mathrm{F} 2$ & $80.3(2)$ \\
\hline $\mathrm{C} 1-\mathrm{C} 2-\mathrm{C} 3-\mathrm{C} 4$ & $-0.5(3)$ & $\mathrm{C} 2-\mathrm{C} 1-\mathrm{C} 8-\mathrm{O} 3$ & $77.8(2)$ \\
\hline $\mathrm{C} 11-\mathrm{C} 2-\mathrm{C} 3-\mathrm{C} 4$ & $-179.22(14)$ & $\mathrm{C} 6-\mathrm{C} 1-\mathrm{C} 8-\mathrm{O} 3$ & $-98.3(2)$ \\
\hline $\mathrm{C} 1-\mathrm{C} 2-\mathrm{C} 3-\mathrm{N} 1$ & $179.37(16)$ & $\mathrm{C} 2-\mathrm{C} 1-\mathrm{C} 8-\mathrm{N} 2$ & $-102.6(2)$ \\
\hline
\end{tabular}




\begin{tabular}{llll}
$\mathrm{C} 11-\mathrm{C} 2-\mathrm{C} 3-\mathrm{N} 1$ & $0.6(3)$ & $\mathrm{C} 6-\mathrm{C} 1-\mathrm{C} 8-\mathrm{N} 2$ & $81.4(2)$ \\
$\mathrm{C} 2-\mathrm{C} 3-\mathrm{C} 4-\mathrm{C} 5$ & $-0.9(3)$ & $\mathrm{N} 2-\mathrm{C} 9-\mathrm{C} 10-\mathrm{C} 11$ & $55.5(3)$ \\
$\mathrm{N} 1-\mathrm{C} 3-\mathrm{C} 4-\mathrm{C} 5$ & $179.25(16)$ & $\mathrm{C} 9-\mathrm{C} 10-\mathrm{C} 11-\mathrm{C} 12$ & $-56.9(3)$ \\
$\mathrm{C} 3-\mathrm{C} 4-\mathrm{C} 5-\mathrm{C} 6$ & $0.9(3)$ & $\mathrm{C} 10-\mathrm{C} 11-\mathrm{C} 12-\mathrm{C} 13$ & $55.8(3)$ \\
$\mathrm{C} 3-\mathrm{C} 4-\mathrm{C} 5-\mathrm{C} 7$ & $-178.87(18)$ & $\mathrm{C} 11-\mathrm{C} 12-\mathrm{C} 13-\mathrm{N} 2$ & $-53.4(2)$ \\
$\mathrm{C} 4-\mathrm{C} 5-\mathrm{C} 6-\mathrm{C} 1$ & $0.5(3)$ & $\mathrm{C} 4-\mathrm{C} 3-\mathrm{N} 1-\mathrm{O} 2$ & $36.6(2)$ \\
$\mathrm{C} 7-\mathrm{C} 5-\mathrm{C} 6-\mathrm{C} 1$ & $-179.75(18)$ & $\mathrm{C} 2-\mathrm{C} 3-\mathrm{N} 1-\mathrm{O} 2$ & $-143.29(19)$ \\
$\mathrm{C} 2-\mathrm{C} 1-\mathrm{C} 6-\mathrm{C} 5$ & $-1.9(3)$ & $\mathrm{C} 4-\mathrm{C} 3-\mathrm{N} 1-\mathrm{O} 1$ & $-141.34(18)$ \\
$\mathrm{C} 8-\mathrm{C} 1-\mathrm{C} 6-\mathrm{C} 5$ & $174.13(18)$ & $\mathrm{C} 2-\mathrm{C} 3-\mathrm{N} 1-\mathrm{O} 1$ & $38.8(3)$ \\
$\mathrm{C} 6-\mathrm{C} 5-\mathrm{C} 7-\mathrm{F} 2^{\prime}$ & $70(2)$ & $\mathrm{O} 3-\mathrm{C} 8-\mathrm{N} 2-\mathrm{C} 13$ & $3.0(3)$ \\
$\mathrm{C} 4-\mathrm{C} 5-\mathrm{C} 7-\mathrm{F} 2^{\prime}$ & $-110(2)$ & $\mathrm{C} 1-\mathrm{C} 8-\mathrm{N} 2-\mathrm{C} 13$ & $-176.62(17)$ \\
$\mathrm{C} 6-\mathrm{C} 5-\mathrm{C} 7-\mathrm{F} 3^{\prime}$ & $-163(2)$ & $\mathrm{O} 3-\mathrm{C} 8-\mathrm{N} 2-\mathrm{C} 9$ & $179.0(2)$ \\
$\mathrm{C} 4-\mathrm{C} 5-\mathrm{C} 7-\mathrm{F} 3^{\prime}$ & $17(2)$ & $\mathrm{C} 1-\mathrm{C} 8-\mathrm{N} 2-\mathrm{C} 9$ & $-0.6(3)$ \\
$\mathrm{C} 6-\mathrm{C} 5-\mathrm{C} 7-\mathrm{F} 1$ & $141.5(2)$ & $\mathrm{C} 12-\mathrm{C} 13-\mathrm{N} 2-\mathrm{C} 8$ & $-130.0(2)$ \\
$\mathrm{C} 4-\mathrm{C} 5-\mathrm{C} 7-\mathrm{F} 1$ & $-38.8(3)$ & $\mathrm{C} 12-\mathrm{C} 13-\mathrm{N} 2-\mathrm{C} 9$ & $53.6(2)$ \\
$\mathrm{C} 6-\mathrm{C} 5-\mathrm{C} 7-\mathrm{F} 3$ & $19.5(3)$ & $\mathrm{C} 10-\mathrm{C} 9-\mathrm{N} 2-\mathrm{C} 8$ & $129.2(2)$ \\
$\mathrm{C} 4-\mathrm{C} 5-\mathrm{C} 7-\mathrm{F} 3$ & $-160.82(18)$ & $\mathrm{C} 10-\mathrm{C} 9-\mathrm{N} 2-\mathrm{C} 13$ & $-54.6(2)$ \\
\hline
\end{tabular}

Hydrogen-bond geometry $\left(\AA,{ }^{\circ}\right)$

\begin{tabular}{lllll}
\hline$D-\mathrm{H} \cdots A$ & $D-\mathrm{H}$ & $\mathrm{H} \cdots A$ & $D \cdots A$ & $D-\mathrm{H} \cdots A$ \\
\hline $\mathrm{C} 6-\mathrm{H} 6 \cdots \mathrm{O}^{\mathrm{i}}$ & 0.95 & 2.59 & $3.526(3)$ & 169 \\
$\mathrm{C} 9-\mathrm{H} 9 A \cdots \mathrm{O} 1^{\mathrm{ii}}$ & 0.99 & 2.45 & $3.361(3)$ & 154 \\
$\mathrm{C} 9-\mathrm{H} 9 B \cdots \mathrm{O} 1^{\mathrm{iii}}$ & 0.99 & 2.58 & $3.369(3)$ & 137 \\
$\mathrm{C} 13-\mathrm{H} 13 A \cdots C g(\mathrm{C} 1-\mathrm{C} 6)^{\mathrm{iv}}$ & 0.99 & 2.92 & $3.447(2)$ & 114 \\
\hline
\end{tabular}

Symmetry codes: (i) $-x+1, y+1 / 2,-z+1 / 2$; (ii) $-x+1 / 2, y+1 / 2, z$; (iii) $x, y+1, z$; (iv) $x+3 / 2,-y+1 / 2,-z$. 